\title{
Antinuclear antibody and HLA-B27 positive uveitis: combination of two diseases?
}

\author{
E H Bosch-Driessen, N M Lardy, A Rothova
}

\begin{abstract}
Aims/background-Anterior uveitis associated with juvenile chronic arthritis concerns two different clinical entities: firstly, antinuclear antibody (ANA) positive patients who have a chronic anterior uveitis with severe complications and often a poor visual prognosis; secondly, usually HLA-B27 positive children, predominantly boys, with unilateral recurrent anterior uveitis. Three patients are described who had a combination of clinical and laboratory features of both diseases.

Methods-Retrospective clinical and laboratory analysis of three patients.

Results-Ocular features in the three patients combined the clinical picture of ANA positive chronic anterior uveitis during early childhood with the clinical features of HLA-B27 unilateral acute anterior uveitis during adolescence. The patients fulfilled the diagnostic criteria of juvenile chronic arthritis, and they had no ankylosing spondylitis. All three patients had the HLA-B $\star 2705$ subtype.

Conclusions-Whether the association of ANA positive chronic anterior uveitis and HLA-B27 unilateral acute anterior uveitis is a coincidence or represents a distinct clinical entity is not yet clear.

(Br F Ophthalmol 1997;81:771-773)
\end{abstract}

Juvenile chronic arthritis (JCA) is not a specific aetiological entity but a term used to describe several diseases, the most important of which are HLA-B27 related spondylarthropathies and juvenile rheumatoid arthritis. ${ }^{1-3}$ Three types of presentation of JCA are known-that is, systemic, polyarticular, and pauciarticular onset. Ocular involvement in JCA (in HLAB27 negative patients) usually consists of bilateral chronic uveitis, which is characterised by many complications and often a poor visual prognosis. ${ }^{1}$ Recently, this was emphasised in a study by Dana et al. ${ }^{4}$ In about $60 \%$ of the patients associated arthritis is of the pauciarticular type; the patients are predominantly women, they have negative rheumatoid factors, but may be positive for antinuclear antibody (ANA) in $71-93 \%$ of cases. ${ }^{1}$ The presence of ANA is a bad prognostic sign for the ocular outcome.

Ocular disease in patients with HLA-B27 positive arthritis or sacroiliitis is characterised by repeated attacks of unilateral, alternating acute anterior uveitis. ${ }^{6}$ This uveitis entity mostly affects male patients and has a favour- able visual outcome. ${ }^{7}$ The rheumatological involvement in childhood mostly affects the peripheral joints; the classic features of ankylosing spondylitis are usually not present.?

\section{Patients and methods}

We describe three patients who had a combination of the clinical and laboratory features of both ANA and HLA-B27 positive uveitis. All patients were examined by a rheumatologist. The criteria described by Bennet and Burch were used for the diagnosis of ankylosing spondylitis. ${ }^{3}$ The diagnosis of JCA was established when the patients fulfilled the EULARWHO criteria. ${ }^{8}$ HLA-B27 typing and subtype distribution was performed in all patients using the polymerase chain reaction (PCR) in combination with the sequence specific oligonucloetide probes (SSOs), described by Lopez-Larrea et al. ${ }^{9}$

PATIENT 1

A 28-year-old male patient had his first ocular examination at the age of 5 years, when he presented with a bilateral visual acuity of finger counting. He suffered from severe bilateral uveitis with synechiae, vitreous opacities, and secondary cataract. Since his third year, his mother had regularly noticed transient mild redness of both eyes. Based on the clinical features and the positive ANA findings, uveitis with JCA was suspected, although there was no joint involvement at that time. Thereafter, he suffered from severe bilateral chronic uveitis and was treated with systemic steroids. At the age of 8 years, band keratopathy and secondary cataract occurred in both eyes. At that time, the visual acuity was hand movements (right eye) and finger counting (left eye). The right eye became hypotonic and the left eye developed secondary glaucoma, for which multiple filtering procedures and cyclodialysis were attempted. From the age of 9 years he had severe episodes of acute unilateral anterior uveitis. The attacks were unilateral, but alternately affected both eyes. His right eye became blind and atrophic, and the left eye developed a cyclitic membrane for which he was treated by a pars plana vitrectomy at the age of 19 years. Thereafter the visual acuity in the left eye improved from finger counting to 0.20 . Since the age of 20 , episodes of unilateral anterior uveitis have been rare and respond well to local corticosteroid therapy. Rheumatological symptoms started at the age of 16 years, when he had arthritis of his knees. At the age of 21 he suffered again from arthralgias of his knees and shoulder, as well as from lower backpain with 
morning stiffness. $x$ Rays of the sacroiliacal joints showed some irregularities, but there was no evidence for the diagnosis of ankylosing spondylitis. The patient is HLA-B27 positive and ANA positive.

PATIENT 2

A 31-year-old female patient was known to have seronegative JCA from the age of 4 years, when she had arthritis of both knees. She was positive for ANA and HLA-B27. Since the age of 12 , she has sufferred from bilateral chronic anterior uveitis. The severity of uveitis required systemic corticosteroid treatment to good effect. At the age of 19 she presented with acute exacerbation of anterior uveitis in her right eye with hypopyon and hypotony. She was treated with periocular and systemic corticosteroids. Since then she has had repeated attacks of unilateral acute anterior uveitis, three of these concomitant with hypopyon and hypotony. The attacks lasted 2-4 months, and were treated with periocular corticosteroid injections and occasionally with systemic corticosteroids. At present, her visual acuity is 0.6 in the right and 1.0 in the left eye. Rheumatological disease at adult age exhibited arthritis followed by arthrosis of the left elbow and right wrist. Severe gonarthritis required a total knee arthroplasty at the age of 26 . An $x$ ray of the pelvis showed no evidence of sacroiliitis.

\section{PATIENT 3}

A 36-year-old male patient was known to have severe chronic anterior uveitis of the right eye from the age of 8 years. At that time, he also had arthritis of the right knee and foot. Seronegative JCA was diagnosed. The right eye developed band keratopathy, cataract, and hypotony and his visual acuity decreased to finger counting. Since the age of 12 years, he has suffered from bilateral chronic anterior uveitis. He developed synechiae, secondary cataract, and glaucoma in both eyes. At the age of 27 , during a vacation in Turkey, he had severe gastroenteritis, followed within 1 week by unilateral iridocyclitis and polyarthritis. Since then, he has had repeated attacks of unilateral acute anterior uveitis including an attack of acute anterior uveitis and arthritis after Campylobacter gastroenteritis contracted in China at the age of 33. Recently, his visual acuity was 0.15 in the right eye and 1.0 in the left eye. $\mathrm{He}$ is HLA-B27 and ANA positive, confirmed at the age of 21 . Radiological examination at the age of $27 \mathrm{did}$ not confirm the diagnosis of ankylosing spondylitis.

\section{Results}

Ocular features in our patients combined the clinical picture of ANA positive chronic anterior uveitis in early childhood with clinical features of HLA-B27 associated unilateral attacks of acute anterior uveitis during adolescence (Table 1).

All patients fulfilled the diagnostic criteria of pauciarticular JCA as formulated by the EULAR-WHO criteria ${ }^{3}{ }^{8}$ but they did not meet the criteria for the diagnosis of ankylosing spondylitis.
Table 1 Ocular and rheumatological disease in ANA and HLA-B27 positive uveitis

\begin{tabular}{|c|c|c|c|c|}
\hline & \multicolumn{2}{|l|}{ Uveitis } & \multirow[b]{2}{*}{$\begin{array}{l}\text { Ankylosing } \\
\text { spondylitis }\end{array}$} & \multirow[b]{2}{*}{$\begin{array}{l}\text { Periphera } \\
\text { arthritis }\end{array}$} \\
\hline & $\begin{array}{l}\text { Acute } \\
\text { anterior }\end{array}$ & Chronic & & \\
\hline Childhood & 0 & 3 & 0 & 3 \\
\hline Adolescence & 3 & 0 & 0 & 3 \\
\hline
\end{tabular}

In HLA-B27 subtype distribution we found the HLA-B ${ }^{\star} 2705$ subtype in all three patients.

\section{Discussion}

Anterior uveitis associated with JCA combines two different clinical entities: firstly, ANA positive patients with chronic anterior uveitis and severe complications such as band keratopathy, secondary glaucoma, cataract, and loss of visual acuity; and, secondly, usually older children, predominantly boys, with unilateral recurrent acute anterior uveitis who are HLAB27 positive and suffer from peripheral arthritis. ${ }^{10}$ The patients of the second group are ANA negative and have a good visual prognosis. $^{\text {? }}$

Concerning the ocular and laboratory examinations, our three patients fulfilled the criteria of both entities. As children, they suffered from bilateral chronic uveitis and they had unilateral exacerbations of acute anterior uveitis as young adults.

As far as the rheumatological findings are concerned, our patients fulfilled the criteria for pauciarticular JCA, but none fulfilled those for ankylosing spondylitis. All patients still suffered from active rheumatic disease as adults. In patient 3, arthritis was initiated by severe gastrointestinal infections, a phenomenon reported in HLA-B27 positive patients. ${ }^{11}$ However, the active rheumatic disease in ANA positive JCA at adult age is not characteristic. The clinical features of patients 1 and 3 are in agreement with the diagnosis of the HLA-B27 related arthropathy type of JCA.

The HLA-B27 antigen includes at least nine subtypes of which HLA-B ${ }^{\star} 2701$, HLA$B^{\star} 2702, H L A-B \star 2705$, and HLA-B ${ }^{\star} 2707$ are found in white populations. Various subtypes are known in the group of HLA-B27 associated diseases, $95 \%$ of the patients with ankylosing spondylitis are HLA-B ${ }^{\star} 2705$ positive, ${ }^{12}$ as are our three patients.

The question is whether the association of the two diseases in our patients is a coincidence or whether they represent a specific entity in rheumatic and ocular disease. In our patients, HLA-B27 genotyping did not give the answer to the question whether a specific allele of HLA-B27 is associated with the ocular features. For an exact answer, the ocular features in adolescence and HLA-B27 typing need to be analysed in a large group of JCA patients. Frequently, when the diagnosis of uveitis associated with JCA is made in a child further investigations are not considered. Therefore, it might well be possible that there are a number of patients who have a combination of features of both diseases such as those we have documented here. Further research is indi- 
cated to identify this presumed entity in rheumatic and ocular disease.

1 Kanski JJ. Juvenile arthritis and uveitis. Surv Ophthalmol 1990;34:253-67.

2 Dequeker J, Mardjuadi A. Prognostic factors in juvenile chronic arthritis. $\mathcal{f}$ Rheumatol 1982;9:909-15.

3 Bennet PH, Burch TA. New York symposium on population studies in the rheumatic diseases: new diagnostic criteria. Bull Rheumatol Dis 1967;17:453-8.

4 Dana MR, Merayo-Lloves J, Schaumberg DA, Foster CS Visual outcomes prognosticators in juvenile rheumatoid arthritis-associated uveitis. Ophthalmology 1997;104:23644.

5 Leak AM, Tuallon N, Muller S, Woo P. Study of antibodies to histone and histone synthetic peptides in pauciarticular juvenile chronic arthritis. Br f Rheumatol 1993;32:426-31.

6 Rosenbaum JT. Acute anterior uveitis and spondylarthropathies. Rheumatol Clin North Am 1992;18:143-51.
7 Korner-Stiefbold U, Sauvain MJ, Gerber N, Garweg J, Korner F, Gugler E. Augencomplikationen bei juveniler chronischer arthritis. Klin Monatsbl Augenheilkd 1993;202 269-80.

8 Brewer EJ Jr, Bass J, Baum J, Cassidy JT, Fink C, Jacobs J, et al. Current proposed revision of JRA criteria. Arthritis Rheum 1977;20:195-9.

9 Lopez-Larrea C, Sujirachato K, Mehra NK, Chiewsilp P, Isarangkura D, Kanga U, et al. HLA-B27 subtypes in Asian patients with ankylosing spondylitis. Tissue Antigens 1995; 45:169-76.

10 Nussenblatt RB, Palestine AG. In: Uveitis. Fundamentals and clinical practice. Chicago: Yearbook Medical Publishers, 1989:164-76

11 Veys EM, Mielants H. Enteropathic arthritis, uveitis, Whipple's disease and miscellaneous spondylarthropathies. Curr Opin Rheumatol 1993;5:420-7.

12 Maclean IL, Iqball S, Woo P, Keat ACS, Hughes RA, Kingsley GH, et al. HLA-B27 subtypes in the spondylarthropathies. Clin Exp Immunol 1993;91:214-9. 